\title{
Unstable and limited crack propagation
}

\section{Numerical modelling and parametric analysis}

\author{
Bing Tie - Haithem Adouani - Clotilde Berdin \\ Silvère Cattet
}

Laboratoire de Mécanique des Sols

Structures et Matériaux (CNRS UMR 8579)

Ecole Centrale Paris, F-92295 Châtenay Malabry cedex

\{bing.tie, haithem.adouani, clotilde.berdin\}@ecp.fr

\begin{abstract}
We consider in this paper the unstable and limited extension of brittle cracks when they initiate near or in local brittle zones and extend through them towards tougher material zones. Our aim is to predict their arrest and in this case, one observes the so-called "pop-in" phenomenon. We use the cohesive zone models as constitutive law of brittle cracks, which are numerically modelled using interface-type elements. Non-linear solvers including loading step adaptive strategy are developed, that is necessary to overcome convergence difficulties during the pop-in. Some numerical aspects specifically related to the pop-in modelling are discussed. Parametric studies on CT specimens are carried out to point out the influential numerical, plastic and fracture parameters. As an example, the yield strength mismatch between the local brittle zone and the tougher material plays a very important role on the pop-in phenomenon and makes its modelling dependent upon the element size at the crack-tip.

RÉSUMÉ. Nous étudions l'extension instable des fissures lorsqu'elles s'amorcent dans des zones locales fragiles afin de prédire leur arrêt dû en particulier à des zones plus tenaces. Cette propagation instable et limitée de fissure est associée au phénomène dit de "pop-in». Le modèle de la zone cohésive est adopté pour modéliser les propriétés d'interface des fissures, qui sont numériquement représentées par des éléments finis joints. Des solveurs non linéaires à pas de chargement adaptatif sont développés. En effet, la stratégie du pas de chargement adaptatif se révèle nécessaire pour pallier des difficultés de convergence pendant le pop-in. Quelques aspects numériques spécifiques à la modélisation du pop-in sont discutés. Des études paramétriques de ce phénomène sur des éprouvettes CT sont présentées. Par exemple, la différence de limite d'écoulement plastique entre la zone locale fragile et le métal de base joue un rôle très important sur le pop-in et a comme conséquence de rendre sa modélisation dépendante de la taille d'élément en fond de fissure.
\end{abstract}

KEYWORDS: unstable crack propagation, pop-in, non-linear solver, cohesive zone models.

MOTS-CLÉS : propagation instable de fissure, pop-in, solveur non linéaire, modèles de la zone cohesive.

DOI:10.3166/REMN.17.663-675 (C) 2008 Lavoisier, Paris

REMN - 17/2008. Giens 2007, pages 663 to 675 


\section{Introduction}

In some industrial components, one can observe initiation and unstable extension of brittle cracks, and then their arrest if they propagate in a decreasing field of stress intensity factor, or if the fracture toughness of the material increases along the cracking path. This study aims at predicting with numerical tools such unstable but limited crack extension, due to the existence of local brittle zones (LBZ) near the crack-tip. This phenomenon is called "pop-in", which usually induces a sudden drop of load on the load-displacement curve during the measurement of the fracture toughness of the material presenting such behaviour.

The cohesive zone theory has been proposed in the pioneering works of (Dugdale, 1960; Barrenblatt, 1962). This theory has a regain of interest nowadays, because of its application in numerical methods used for modelling the fracture of materials (see in Chandra et al., 2002). A cohesive zone model (CZM) combines an energy criterion with a local critical stress criterion in order to express dissipative mechanisms in the fracture process zone (FPZ) and lump this zone into the so-called "cohesive crack surfaces" by introducing a surface fracture energy. By choosing appropriate fracture parameters associated to a cohesive law, brittle crack as well as ductile crack extension can be modelled. Different from both the global and the local approaches of fracture, CZM constitutes therefore an alternative way to model crack propagation.

The use of CZM requires explicit modelling the cohesive crack surfaces. The two most popular numerical methods to create the cohesive surfaces are the XFEM (eXtended Finite Element Method) (Moës et al., 2002; de Borst, 2003), or the use of the interface-type finite elements (Xu et al., 1994; Camacho et al., 1996). In this work, the second method is adopted: 2D and 3D interface-type finite elements are implemented in our object oriented finite element code OOFE (Object Oriented Finite Element program) and validated.

In this study, the pop-in phenomenon due to a local brittle zone that is present ahead of the crack tip is studied. The local brittle zone is taken into account by a fracture behaviour different from the base material but also by a different mechanical behaviour. So, the triggering of a brittle crack followed by its propagation through two materials has to be modelled.

Since brittle crack propagation generally occurs with a high speed, the choice of static analysis is questionable. Nevertheless, ASTM (1999) procedure is based on a static analysis. Furthermore, a limited duration of unstable crack propagation is expected so that the wave reflection could have been neglected. Hence, the kinetic energy released by the brittle extension of the crack is neglected in a first step and the analyses presented herein have been performed under the static hypothesis. Dynamic analysis is being performed by our current work but is not presented here (Adouani et al., 2006, 2007).

The pop-in phenomenon is highly non-linear and results in convergence difficulties for the classical qNR (quasi Newton-Raphson) solvers. This point is 
discussed and an appropriate adaptive strategy of loading step is proposed. Although the cohesive zone model is known to avoid mesh size effect if the mesh size is sufficiently small (Chaboche et al., 2001), the influence of the element size on the pop-in modelling is studied because it appeared in the simulations. Such element size dependence seems to be a specific feature of the pop-in modelling.

Finally, a parametric study on a compact tensile (CT) specimen is performed in order to point out the influential material parameters of the pop-in modelling among the mechanical behaviour and the fracture parameters.

\section{Model problem within the CZM frameworks}

This section summarizes the governing equations of the model problem and several basic aspects of the well-known CZM used to model the crack propagation under static conditions.

We consider the static equilibrium of a body $\Omega$ with a crack $\Gamma_{c_{0}}$, which is submitted to body forces $\boldsymbol{f}$ and to boundary conditions along its outer surface $\partial \Omega$ : the prescribed forces $\boldsymbol{g}$ on $\Gamma_{\sigma} \subset \partial \Omega$ and the prescribed displacements $\boldsymbol{u}_{d}$ on $\Gamma_{u} \subset \partial \Omega$ (Figure 1a). The extension $\Gamma_{c}(t)$ of the crack $\Gamma_{c_{0}}$ has to be solved and $\Gamma_{c}(0)=\Gamma_{c_{0}}$. In the static case, $t$ denotes the dependence of $\Gamma_{c}(t)$ upon external loading history. According to the cohesive zone theory, the fracture process zone surrounding the crack tip is lumped into the cohesive surface $\Gamma_{c}$. The energy dissipated during the crack propagation in the FPZ is taken into account by a cohesive constitutive law, which determines the cohesive traction vector $\boldsymbol{T}^{ \pm}$applied on the two lips $\Gamma_{c}^{ \pm}$of the crack $\Gamma_{c}$ as function of the displacement jump $[\boldsymbol{u}]_{x}$ between them. The equilibrium equations and boundary conditions read as follows:

$$
\begin{array}{ll}
\operatorname{Div}(\sigma(x))+\boldsymbol{f}=\boldsymbol{0} & \text { in } \Omega \\
\sigma(\boldsymbol{x}) \cdot \boldsymbol{n}=\boldsymbol{g} & \text { on } \Gamma_{\sigma} \\
\sigma(\boldsymbol{x}) \cdot \boldsymbol{n}^{ \pm}=\boldsymbol{T}^{ \pm}, \boldsymbol{T}=\boldsymbol{T}^{-}=-\boldsymbol{T}^{+} & \text {on } \Gamma_{\boldsymbol{c}}^{ \pm} \\
\boldsymbol{u}=\boldsymbol{u}_{d} & \text { on } \Gamma_{\boldsymbol{u}} \\
{[\boldsymbol{u}]_{\boldsymbol{x}} \cdot \boldsymbol{n}^{-} \geq 0} & \text { on } \Gamma_{\boldsymbol{c}}
\end{array}
$$

where $\boldsymbol{\sigma}$ is the Cauchy stress tensor, $\boldsymbol{n}$ and $\boldsymbol{n}^{ \pm}$the outward unit normal vectors on $\Gamma_{\sigma}$ and $\Gamma_{c}^{ \pm}$respectively. 
The constitutive law for the mechanical behaviour of the bulk material in the domain $\Omega$ is a classical plasticity law - the von Mises one with isotropic hardening, whereas a cohesive law is used for the cohesive surface $\Gamma_{c}$ :

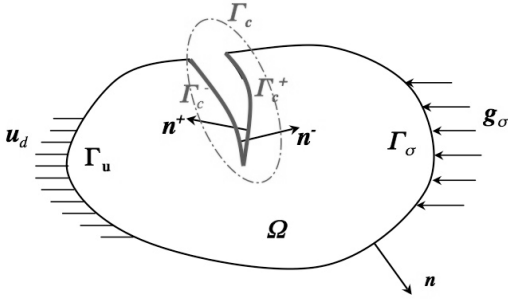

a)

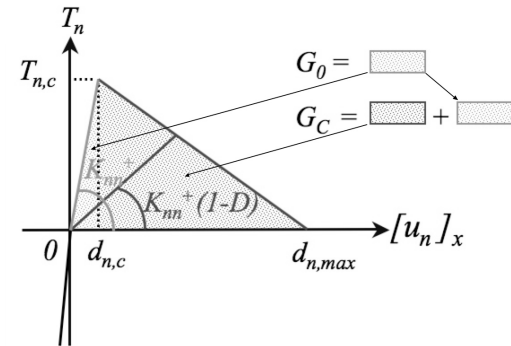

b)

Figure 1. (a) Definition domain $\Omega$ with a crack $\Gamma_{c}$; (b) Cohesive zone law (Crisfield et al., 1997)

$$
\begin{aligned}
& \partial_{\boldsymbol{t}} \boldsymbol{\sigma}(\boldsymbol{u})=\boldsymbol{C}^{e p}(\boldsymbol{\sigma}, \boldsymbol{R}): \varepsilon\left(\partial_{\boldsymbol{t}} \boldsymbol{u}\right) \quad \text { in } \Omega \\
& \boldsymbol{T}\left([\boldsymbol{u}]_{x}\right)=\left(1-D\left([\boldsymbol{u}]_{x}\right)\right) \boldsymbol{K}^{+} \cdot[\boldsymbol{u}]_{x} \quad \text { on } \Gamma_{\boldsymbol{c}}
\end{aligned}
$$

where $\varepsilon$ is the infinitesimal strain tensor, $\boldsymbol{R}$ the hardening variable, $\boldsymbol{C}^{e p}$ the elasticplastic tensor, $D \in[0,1]$ a damage variable and $\boldsymbol{K}^{+}$is a positive definite matrix of (dummy) stiffness parameters of the elastic regime of a cohesive law. This elastic regime prior to the onset of damage is usually introduced for numerical concerns and results in physically meaningless deformations in the cohesive interface during the pre-cracking phase. Fortunately one can largely reduce these unwanted deformations by choosing sufficiently large values of the parameters of $\boldsymbol{K}^{+}$.

As far as the evolution law of the damage variable $D\left([\boldsymbol{u}]_{x}\right)$ is concerned, a damage potential $\Phi(G)$ can be defined in different ways and results in different shapes of the cohesive traction-displacement jump relation (Valoroso et al., 2006):

$$
\begin{aligned}
& \Phi(G)=G\left([\boldsymbol{u}]_{x}\right)-G^{*}\left(D\left([\boldsymbol{u}]_{x}\right)\right) \\
& G\left([\boldsymbol{u}]_{x}\right)=\frac{1}{2} K_{n n}^{+} \frac{<\left[u_{n}\right]_{x}>_{+}^{2}}{d_{n, c}}
\end{aligned}
$$

In Equation [3b], only the normal displacement jump $\left\langle\left[u_{n}\right]_{x}\right\rangle_{+}$across the cohesive crack $\Gamma_{c}$ appears, as only the opening mode of crack propagation is considered here. $K_{n n}^{+}$is the parameter in $\boldsymbol{K}^{+}$, which is associated to the opening mode and $d_{n, c}$ is the 
critical displacement opening jump that delimits the pre-cracking elastic regime from the material degrading one.

In our work, the following choice of $G^{*}(D)$ is adopted, which leads to the wellknown bilinear cohesive model proposed by Crisfield (Crisfield et al., 1997):

$$
G^{*}(D)= \begin{cases}\frac{G_{c}^{2} G_{0}}{\left.\operatorname{lG}_{0} D+G_{c}(1-D)\right]^{2}} & \text { if } D \in[0,1[ \\ \underset{t \in[0, T]}{\max G(t)} & \text { if } D=1\end{cases}
$$

where $G_{c}$, formally defined as $\int T_{n} d u$, is the fracture energy, $G_{0}$ is the part of $G_{c}$ associated to the elastic regime, $T_{n, c}$ is the tensile strength and $d_{n, \max }$ the maximal opening jump at the fully damaged state $(D=1)$ (Figure 1b). The fracture energy $G_{c}$ can differ from the global fracture parameter because of plastic dissipation (Siegmund et al., 1997). Hence, the fracture is controlled by two criteria: a critical stress criterion with $T_{n, c}$ and an energetic one with $G_{c}$. To get a reliable prediction of the crack extension, an appropriate choice of the fracture parameters $\left(T_{n, c}, d_{n, \max }, G_{c}\right)$ is of utmost importance.

\section{Several numerical aspects of the pop-in modelling}

To predict the pop-in phenomenon during a fracture toughness test, a CT type specimen is chosen. A small zone is introduced in the pre-cracked specimen in front of the crack tip. This zone has a mechanical and fracture behaviour different from the rest of the specimen in order to model the pop-in phenomenon coming from a brittle zone: The flow stress of the local brittle zone is higher than the one of the surrounding material, its critical stress is lower than the one of the surrounding material, whereas the fracture energies are equivalent. The local brittle zone extends on $9 \%$ of the ligament length in order to lead to a significant pop-in in terms of ASTM analysis (ASTM, 1999). As the local brittle zone is supposed to be present all along the crack front, 2D plane strain analyses have been performed, as well as 3D analyses.

In our numerical modelling of crack propagation, the cohesive surface is discretised by the interface-type finite elements. In the present work, the path of the crack propagation is assumed to remain along a symmetry plane, so the interfacetype cohesive elements are initially introduced on this path. A sufficiently fine mesh is used near the crack tip, in order to solve the mechanical fields in front of the crack tip (Figure 2a). Different mesh sizes around the crack propagation path have been used in order to analyse their influence. No adaptive remeshing is used in this work. 


\subsection{Loading step adaptive non-linear solvers}

When the onset of cracking occurs in or near a local brittle zone and propagates through it toward tougher material, an instable but limited extension of crack can be observed and results in the pop-in phenomenon expressed by a sudden drop of load on the load-displacement curve (Figure 2b). The pop-in phenomenon is highly non linear and generally causes difficulties to non-linear solvers:

- to capture the beginning of the load drop at the onset of the pop-in, and

- to correctly recover the load-displacement curve in the tougher material at the end of the pop-in.

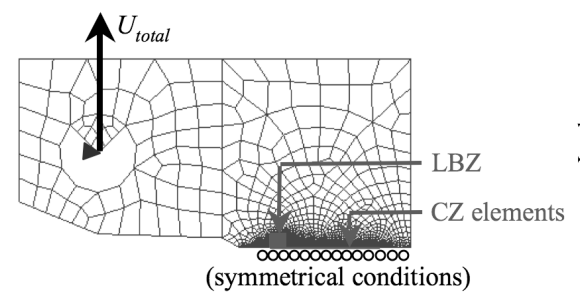

a)

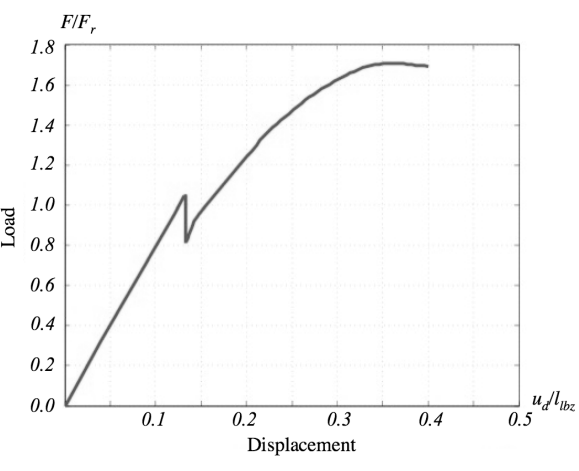

b)

Figure 2. The pop-in phenomenon in a CT specimen due to a local brittle zone (the red rectangular zone): (a) finite element mesh of the half of the CT specimen; (b) the load-displacement curve indicates a sudden and brief load drop

In the present work, a qNR solver is developed and tested. It was at first implemented in its most economic version, i.e. the tangent matrix is calculated, factorized and stored once at the beginning of the first loading step. Hence, we use the elastic stiffness matrix of the initial structure as iterative matrix for the non-linear solving of all loading steps. To improve the robustness of this qNR solver during the pop-in phenomenon, two ingredients seem to be essential.

The first ingredient is a loading step adaptive strategy that allows not only the decrease of the loading step prior to and during the pop-in but also its increase at the end of the pop-in. Indeed, prior to the beginning of the pop-in, a sufficiently small loading step is necessary to capture the beginning of the load drop and to avoid the underestimation of the maximal load reached before the pop-in. Next, during the pop-in, a very small loading step is necessary to guarantee the convergence. In our numerical modelling of CT specimens with prescribed displacement $U_{\text {total }}$, the 
loading step generally falls to $\Delta U=10^{-11} U_{\text {total }}$ when the pop-in occurs. However, if such small loading step is remained after the pop-in, oscillating phenomena can be observed on the load-displacement curve (Figure 3a). Such numerical oscillations can be removed by adaptively enlarging the loading step after the pop-in (Figure 3b).

The second ingredient is the update of the tangent matrix of the non-linear solver. Even if the numerical oscillations after the pop-in are removed (Figure $4 b$ ), it is obvious that the load-displacement curve after pop-in is perturbed because the response of the structure containing now a longer crack is iteratively resolved using its initial stiffness matrix as tangent operator, which corresponds to a shorter crack. Hence, when the tangent matrix is updated at the beginning of each loading step, we get finally a load-displacement curve without spurious numerical perturbations (Figure 2b). Finally, to combine the accuracy, the robustness and the performance, this update is only necessary and done at the end of the pop-in.

We note that, in some loading cases, once the onset of the pop-in is captured, an arclength type algorithm such that the one proposed by (Riks et al., 1996; Crisfield et al., 1997) should improve the performance and the robustness of non linear solvers during the pop-in.

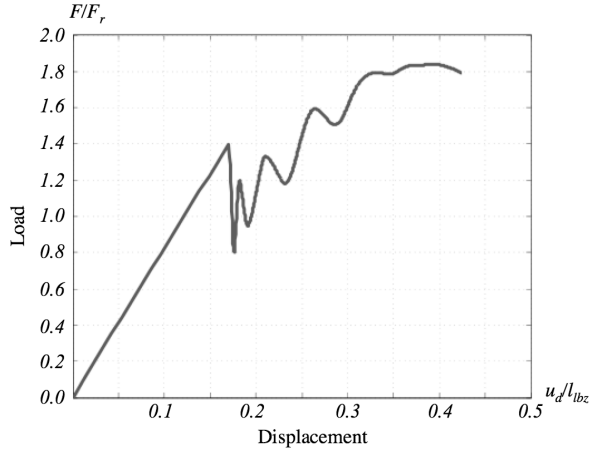

a)

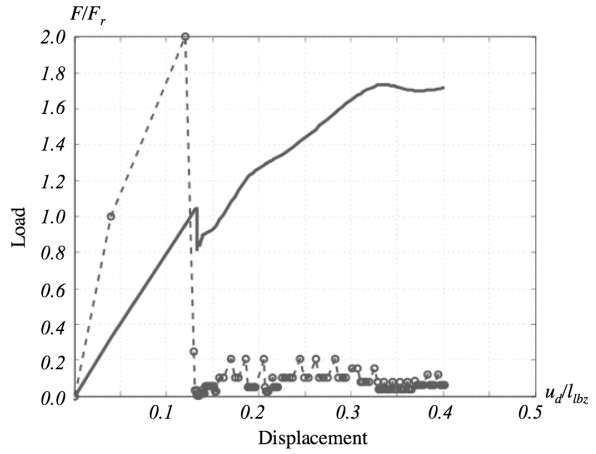

b)

Figure 3. Load-displacement curves showing: (a) oscillating phenomena at the end of the pop-in due to very small loading step; (b) oscillation elimination with the adaptive strategy of loading steps and (b) the evolution of the loading step

\subsection{Influence of numerical parameters}

As far as the choice of the numerical parameters $\left(K_{n n}^{+}, d_{n, c}, G_{0}\right)$ of the cohesive law is concerned, it is necessary to take a small enough $d_{n, c}$, which results in a small 
enough $G_{0}$ or a large enough $K_{n n}^{+}$, so that the unwanted reversible deformations during the pre-cracking phase remain insignificant. Of course, the smaller is the value of $d_{n, c}$, the more difficult is the convergence. However, for the static modelling of pop-in phenomena, the qNR solver is robust enough to overcome convergence difficulties due to small values of $d_{n, c}$.

Another important numerical parameter to analyse is the size of cohesive interface elements and volume elements near the crack-tip (Chaboche et al., 2001). One criterion of choice of the element size at the crack-tip comes from the following estimated length $l_{c z}$ of the cohesive zone, where the damage variable $D$ evolves from 0 to 1 (Falk et al., 2001):

$$
l_{c z}=\frac{9 \pi}{32} \frac{E}{1-v^{2}} \frac{2 G_{c}}{T_{n, c}^{2}}
$$

Obviously, the element size should be smaller than $l_{c z}$ so that there are several elements in the cohesive zone. In the case of a CT specimen with a local brittle zone at the crack-tip (Figure 2a), if we denote $h$ the element size that has been identified for some particular material according to its grain size, to $l_{c z}$ and by comparison between experimental data and numerical modelling, several computations have been performed using respectively the following element sizes around the crack-tip: $0.125 h, 0.25 h, 0.5 h, h, 2 h$ and $4 h$. It can be observed that: the smaller the element size, the later the onset of the pop-in (Figure 4a) and there is no convergence observed when the element size decreases.

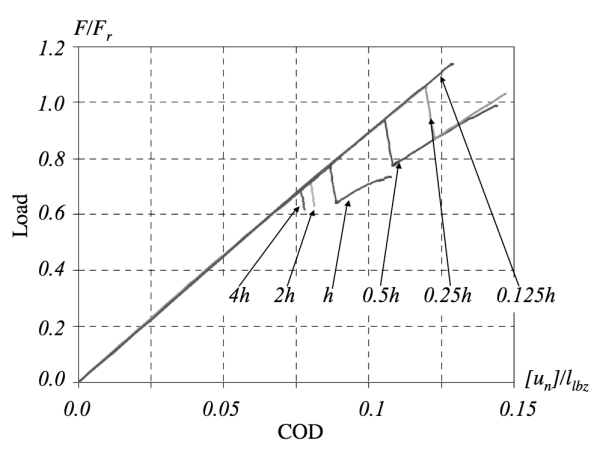

a)

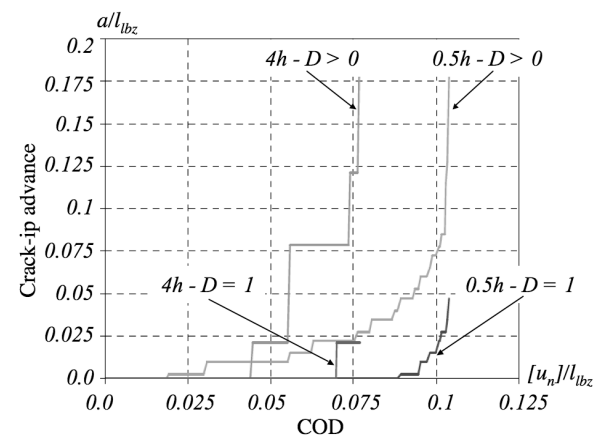

b)

Figure 4. Influence of the element size near the crack-tip on the onset of the pop-in in a CT specimen with a LBZ, both the LBZ and the surrounding material are elastic-plastic and for the cohesive law, $G_{c} \gg G_{0}$. (a) Load-COD curves observed using different element sizes: $0.125 \mathrm{~h}, 0.25 \mathrm{~h}, 0.5 \mathrm{~h}, \mathrm{~h}, 2 \mathrm{~h}$ and $4 \mathrm{~h}$; (b) Advance of damaging crack-tip $(D>0)$ and the broken crack-tip $(D \geq 1)$ 
To understand this element size dependence, others analyses have also been carried out leading to the following remarks:

- as mentioned above, the cohesive law controls the crack extension by two criteria. The first one is based on the critical stress, which is reached more rapidly if the element size is smaller, so the advance of the damaging crack-tip $(D>0)$ initiates more rapidly with smaller element size (Figure $4 \mathrm{~b}$ ). The second criterion is based on the fracture energy $G_{c}$ and we observe that, the bigger is the element size, the faster is the $G_{c}$ dissipation in the cohesive crack interfaces, the advance of the broken crack-tip $(D=1)$ and so the onset of the pop-in. Such element-size dependence is only observed when the LBZ has a plastic behaviour different from the one of the surrounding material;

- in elastic case, there is no element-size dependence on the onset of the pop-in, hence, the element size seems to have great influence on the evaluation of plasticity developing around the crack-tip;

- in the case where $G_{c}=G_{0}$, so the initiation of cracking is essentially controlled by the criterion based on a critical stress $T_{n, c}$, the dependence on the element size observed agrees with something well-known in numerical modelling of stress around a crack-tip: the smaller the element size, the higher the stresses evaluated near the crack-tip, so the earlier the onset of the pop-in (Figure 5a);

- in the case of plasticity and $G_{c}>G_{0}$, the mesh size dependence is the consequence of the material heterogeneity introduced by the LBZ, due to the mismatch of yield strength between the LBZ and the surrounding tougher material (Figure 5b).

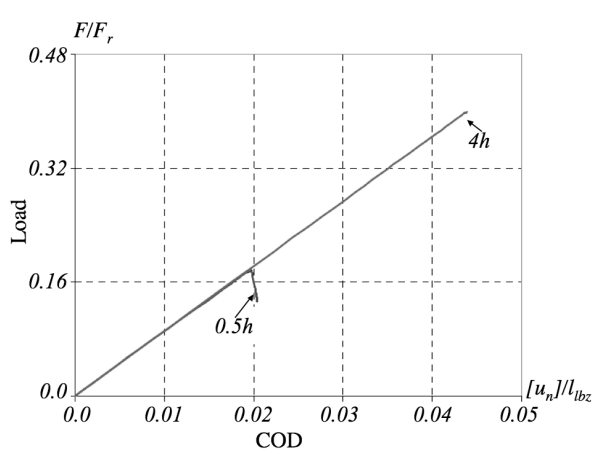

a)

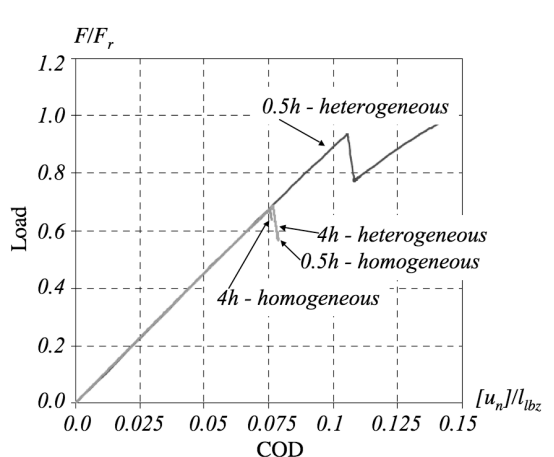

b)

Figure 5. Influence of the element size around the crack-tip on the onset of the popin, Load-Crack Opening Displacement (COD) curves observed in the cases where: (a) $G_{c}=G_{0}$ for the cohesive law; (b) the CT specimen is homogenous or has a LBZ with a yield strength different from the one of the surrounding material 
Hence, the mesh size dependence seems to be a particular aspect of the numerical modelling of the pop-in using the cohesive zone models, because the local brittle zone and the mismatch of yield strength is the origin of the pop-in phenomenon. Further analyses should be carried out in order to understand the way the plasticity and the energy dissipated in the fracture process zone develop and interact in the narrow heterogeneous zone around the crack-tip and to clarify how this interaction results in the observed dependence of the element size.

\section{Influential plasticity and fracture parameters of the pop-in phenomenon}

To get correct prediction of the unstable and limited crack extension during the pop-in phenomenon, appropriate choices of plastic and fracture parameters are of the utmost importance. Parametric studies have been carried out and give the following remarks.

Among the plastic parameters, we note that:

- in the case where the hardening law is obtained from the uniaxial tension test, the way of interpreting the Lüders plateau influences the onset of the pop-in (Figure 6a);

- the yield strength mismatch between the LBZ and the surrounding tougher material influences the onset of the pop-in. The higher is the yield strength of the LBZ, the larger the mismatch, the earlier the onset of pop-in (Figure 6b).

But it is important to underline that in these two cases, the crack extension during the pop-in is not notably influenced by these plastic parameters. Generally, it has been observed that the larger the load at the pop-in triggering, the larger the drop of the load. This is due to a larger energy released in the structure.

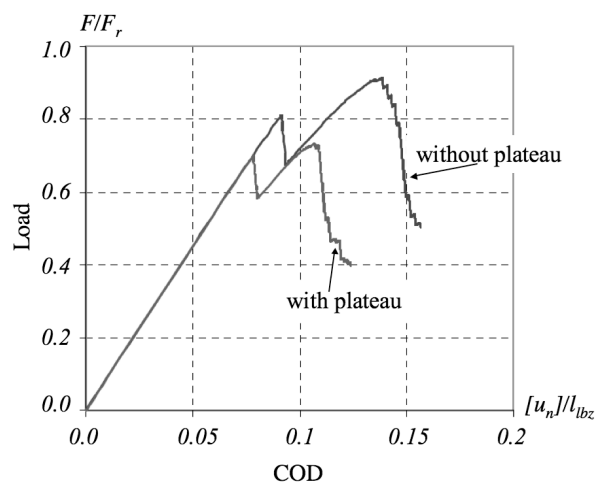

a)

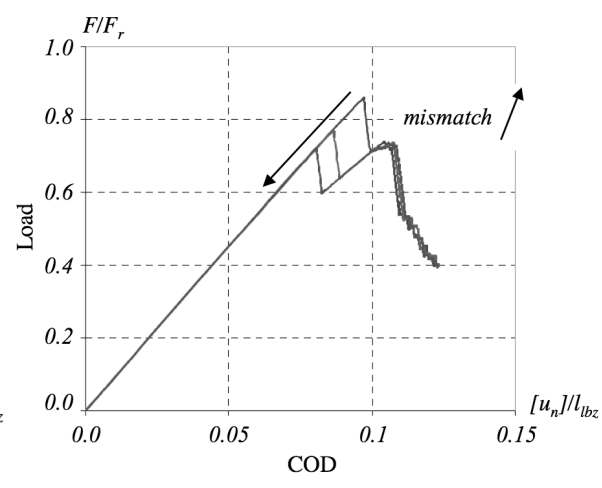

b)

Figure 6. Influence of: (a) the interpretation of the Lüders plateau and; (b) the mismatch of yield strength between the LBZ and the tougher material 
Among the fracture parameters, we note that:

- the critical stress $T_{n, c}$ of the cohesive elements locating in front of the crack-tip over a small length $l_{i}$ influences in a important way both the onset of the pop-in and the crack extension during it;

- the fracture energy $G_{c}$ of the LBZ influences both the onset of pop-in and the crack extension at the two stages of crack initiation and propagation.

The location of the LBZ about the crack-tip has also great importance for the pop-in phenomenon. Three locations of the LBZ have been modelled as well as the two homogeneous cases where there is only the tougher material or only the brittle material (Figure 7a). It is found that the most critical situation is when the LBZ locates in front of the crack-tip, because we get a bigger pop-in. Indeed, as the onset of the pop-in is delayed, so the maximal load reached before the pop-in increases, so the load drop and the released energy in the structure are more important.

Otherwise, the 3D effect at the crack-tip should to be taken into account (Figure 7b). As finite elements around the crack-tip should be of sufficiently small size, it is very expensive even prohibitive to perform a whole $3 \mathrm{D}$ modelling. So, a mixed 2D-3D modelling is interesting and allows taking into account 3D effects, such as a curved crack front, in numerical computing (Figure 8).

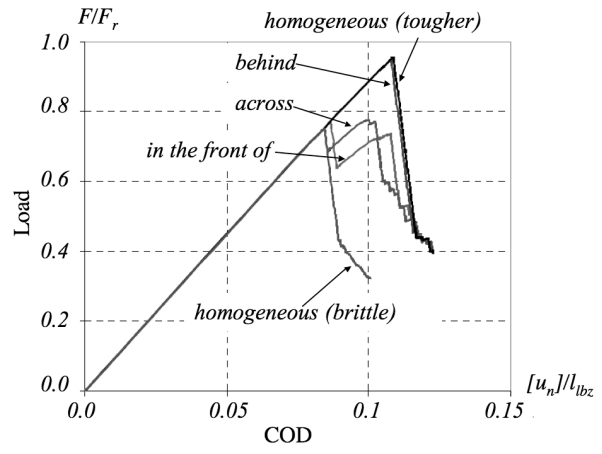

a)

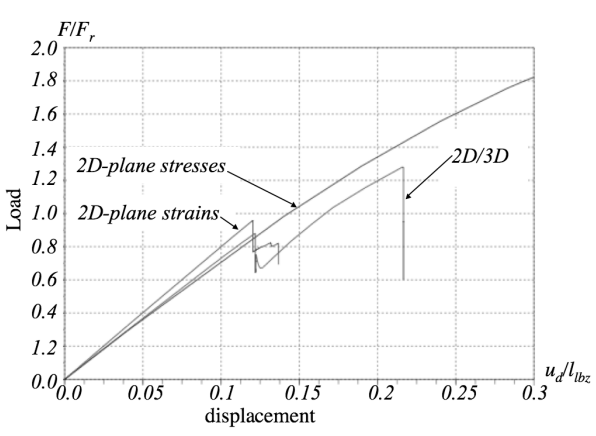

b)

Figure 7. (a) Influence of the location of the LBZ about the crack-tip; (b) $3 D$ effect at the crack-tip on the pop-in phenomenon 


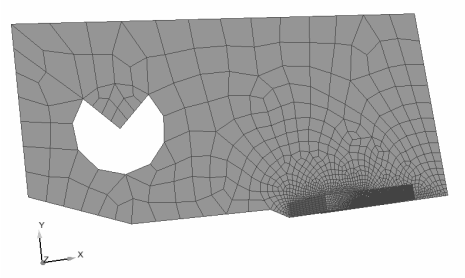

a)

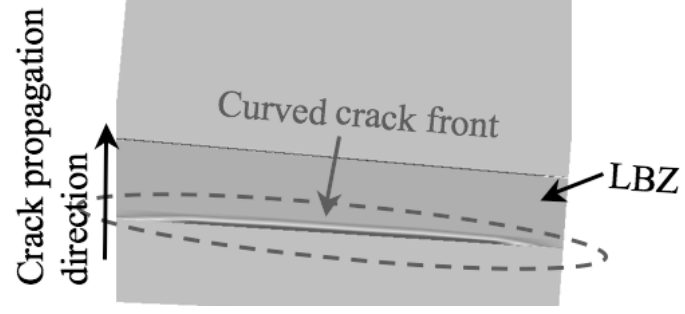

b)

Figure 8. (a) Mixed $2 D / 3 D$ mesh of the half CT specimen; (b) curved crack advancing front

\section{Conclusion}

The numerical modelling of unstable and limited brittle crack propagations - the pop-in phenomenon - is considered here. Particular numerical aspects are discussed such as the loading step adaptive strategy and the dependence on the element size at the crack-tip. Due to the loading step adaptive strategy, the developed quasiNewton-Raphson non-linear solver is sufficiently robust to capture the onset and the end of the pop-in and give correct prediction of crack extensions. But it would be interesting to compare this solver to a Riks-type algorithm. The dependence on the element size is probably a particular aspect of the pop-in modelling, because it is only observed when there is yield strength mismatch between the local brittle zone and the surrounding tougher material. More detailed analyses are currently done in order to understand this dependence.

Numerical analyses on CT specimens are carried out and point out the influential mechanical and fracture parameters of the pop-in phenomenon, especially the tensile strength over a characteristic length in front of the crack-tip and, the fracture energy, and the mismatch of yield strength between the local brittle zone and the surrounding tougher material. The location of the local brittle zone has a great influence on the pop-in too: generally, the later the onset of the pop-in, the larger the pop-in load, the bigger the pop-in and the greater the load drop and the crack extension. Finally, it is very important to make 3D modelling around the cracking path due to the important $3 \mathrm{D}$ effect on the crack propagation. 


\section{References}

Adouani H., Tie B., Berdin C., Aubry D., "Adaptive numerical modelling of dynamic crack propagation", $8^{\text {th }}$ International conference on mechanical and physical: Behavior of materials under dynamic loading, September 2006, Dijon, France.

Adouani H., Tie B., Berdin C., « Modélisation numérique de la propagation instable et limitée de fissure due à la présence de zones locales fragiles ", $8^{e}$ Colloque National en Calcul des Structures, Mai 2007, Giens, France.

ASTM 1999, Standard test method for measurement of fracture toughness, ASTM E 182099.

Barrenblatt G. I, "The mathematical theory of equilibrium of cracks in brittle fracture", $A d v$. Appl. Mech., 7, 1962, p. 55-129.

Borst R. de, "Numerical aspects of cohesive zone models", Engineering Fracture Mechanics, 70, 2003, p. 1743-1757.

Camacho G.T., Ortiz M., "Computational modeling of impact damage in brittle materials", International Journal of Solids and Structures, 33, 1996, p. 2899-2938.

Chaboche J.L., Feyel F., Monerie Y., "Interface debonding models: a viscous regularization with a limited rate dependency", International Journal of Solids and Structures, 38, 2001, p. $3127-3160$.

Chandra N., Li H., Shet C., Ghonem H., "Some issues in the application of cohesive zone models for metal-ceramic interfaces", International Journal of Solids and Structures, 39, 2002, p. 2827-2855.

Crisfield M. A., Jelenic G., Mi Y., Zhong H.-G., Fan Z., "Some aspects of the non-linear finite element method", Finite Elements in Analysis and Design, 27, 1997, p. 19-40.

Dugdale D. S., "Yielding of steel sheets containing slits", J. Mech. Phys. Solids, 8, 1960, p. $100-104$

Falk M.-L., Needleman A., Rice J.-R., "A critical evaluation of cohesive zone models of dynamic fracture", $5^{\text {th }}$ European Mecahnics of Materials Conference, March 2001, Delft, Netherlands, Journal de Physique IV France, vol. 11, p. Pr5-43-50.

Moës N., Belytschko T., "Extended finite element method for cohesive crack growth", Engng. Fract Mech, 2002, 69, p. 813-33.

Riks E., Rankin C., Brogan F.A., "On the solution of mode jumping phenomena in thinwalled shell structures", Comp. Meth. Appl. Mech. Eng., 136, 1996, p. 54-92.

Siegmund T., Needleman A., "A numerical study on dynamic crack growth in elasticviscoplastic solids”, Int. J. Solids Struct., 43, 1997, p. 769-787.

Valoroso N., Champaney L., "A damage-mechanics-based approach for modelling decohesion in adhesively bonded assembles”, Eng. Frac. Mech., 73, 2006, p. 2774-2801.

$\mathrm{Xu}$ X.P., Needleman A., "Numerical simulations of fast crack growth in brittle solids", Journal of the Mechanics and Physics of Solids, 42, 1994, p. 1397-1434. 
